\title{
Kinetics of Methane Hydrate Formation in an Aqueous Solution with and without Kinetic Promoter (SDS) by Spray Reactor
}

\author{
Yaqin Tian, ${ }^{1,2,3}$ Yugui Li, ${ }^{1,2,3}$ Hongping An, ${ }^{1,3}$ Jie Ren, ${ }^{1,3}$ and Jianfeng Su ${ }^{1,2}$ \\ ${ }^{1}$ College of Materials Science and Engineering, Taiyuan University of Science and Technology, Taiyuan 030024, China \\ ${ }^{2}$ Shanxi Provincial Key Laboratory of Metallurgical Device Design Theory and Technology, \\ Taiyuan University of Science and Technology, Taiyuan 030024, China \\ ${ }^{3}$ Collaborative Innovation Center of Taiyuan Heavy Machinery Equipment, \\ Taiyuan University of Science and Technology, Taiyuan 030024, China
}

Correspondence should be addressed to Yaqin Tian; tianyaqin203@163.com

Received 21 June 2017; Accepted 27 July 2017; Published 6 September 2017

Academic Editor: Barbara Gawdzik

Copyright (C) 2017 Yaqin Tian et al. This is an open access article distributed under the Creative Commons Attribution License, which permits unrestricted use, distribution, and reproduction in any medium, provided the original work is properly cited.

Hydrate formation apparatus reported so far was mainly concentrated in stirred-tank batch environments. It was difficult to produce the high gas storage hydrate efficiently. Some nonstirred technology has been attracting more attention by researchers. This work proposed a new apparatus for hydrate formation by spraying water into a gaseous phase with a fine nozzle. It can get sufficient contact surface area for gas-liquid reaction. Methane hydrate formation experiments have been conducted using pure water and sodium dodecyl sulfate (SDS) aqueous solution for comparison at $277.15 \mathrm{~K}$. The experiments were conducted at 7.0 and $6.0 \mathrm{MPa}$, respectively. Kinetics of methane hydrate formation have been investigated by methane consumption per mole of water and reaction rate. The mechanism of hydrate formation and kinetics property by spraying atomization were studied with the theory of crystal chemistry.

\section{Introduction}

Gas hydrates are nonstoichiometric crystal structures that can trap guest molecules in the well-defined host lattices built up from hydrogen-bonded water molecules. There are three distinct crystal types: structure I (sI), structure II (sII), and structure $\mathrm{H}(\mathrm{sH})$, which differ in sizes and shapes of the cages formed [1]. It has long been known that gas hydrates possess the remarkable capacity to compress a large volume of gas into a relatively small volume of hydrate where a commonly quoted number as $1 \mathrm{~m}^{3}$ of hydrate, at standard conditions, can contain up to $170 \mathrm{~m}^{3}$ of natural gas [2].

Recently, there have been numerous proposals to make use of gas hydrates in water desalination, $\mathrm{CO}_{2}$ capture and sequestration [3-5], food industry, refrigeration, gas separation [6,7], and gas storage and transportation [8]. However, gas hydrates based technology requires the ability to be formed in a rapid and predictable manner (i.e., kinetics) [9]. There are two approaches to overcome the slow kinetics of hydrate formation: the first approach is via the use of kinetic promoters to enhance the kinetics [10] and the second approach is via the use of innovative gas/liquid contact modes [11].

During the gas hydrate formation methane is generally less soluble in water. In the case of no disturbance, few hydrates are generated only at the gas-liquid interface [12]. Although the ideal hydrate formation rate can be obtained by mechanical stirring, the mechanical reliability problems caused by high-pressure mixing and liquid viscosity increased in the later period of the formation of gas hydrate [13]. This will affect the efficiency of mechanical stirring and then reduce the gas storage rate of unit volume [14]. Therefore, a new efficient method for high gas storage hydrate formation is urgently needed. The hydrate formation is the physical and chemical reaction of the gas and liquid which is easy to generate gas hydrate in the gas-liquid interface. According to the principle of chemical reaction kinetics, the increase of the number of the interfaces is very important 


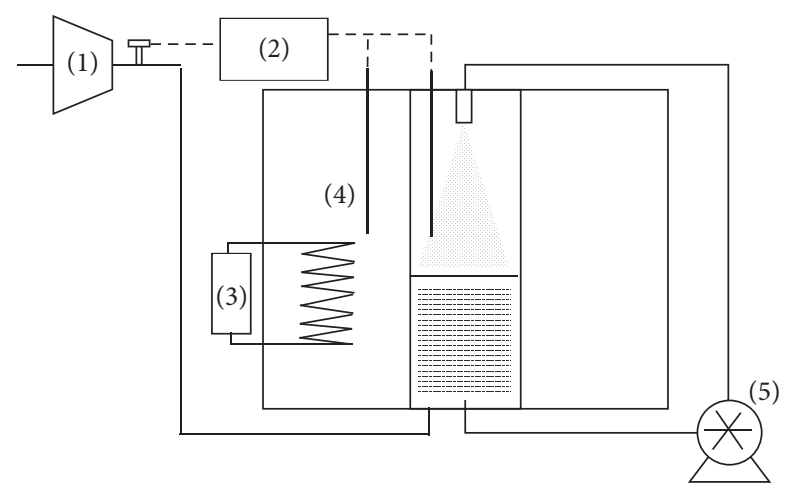

(1) Gas supply system

(2) Data display gathering system

(3) Refrigeration system

(4) Bath trough

(5) Circulating pump

FIGURE 1: Schematic diagram of forming hydrates in spray reactor.

for accelerating the reaction [15]. So, the spray method can produce high gas storage hydrate quickly. This method is efficient and simple and can meet the requirements of the experiment and production [16].

In this study, methane hydrate formation experiments have been conducted with pure water and aqueous solution of sodium dodecyl sulfate (SDS) at 277.15 K. Methane consumption per mole of water and reaction rate have been reported in the spray reactor with SDS aqueous solution. Kinetics of methane hydrate formation for pure water and SDS aqueous systems have been investigated at 7.0 and $6.0 \mathrm{MPa}$.

\section{Experiment}

The experimental apparatus was shown in Figure 1. The device consisted of a gas supply system, a control display system, a refrigeration system, and a circulating reaction system. In the gas supply system (1), the experimental gas flowed out of the cylinder through the valve to the buffer tank. Then the pressure of the gas was controlled at the desired fixed value by the pressure regulator. Finally, the gas entered the reactor for hydrate formation. Pressure and temperature of the reactor were controlled and recorded by the system of the control system (2). Refrigeration system (3) was kept at a constant temperature of bath by cooling medium through the coil. The water or solution in the reaction was directly added to the reactor before the reaction. In the reaction process, the solution was pumped to the top of the nozzle cycle by the circulating pump (5). The experimental data was automatically detected and recorded by the control display system (2).

2.1. Instrument and Equipment. The formation of gas hydrate is a high-pressure and low-temperature reaction. In the process of reaction, interface of gas-liquid needs to be disturbed, until the formation of ice crystal gas hydrate. In this paper, the effective volume of the reactor was $14 \mathrm{~L}$; the maximum working pressure was $35 \mathrm{MPa}$. The reactor consists of a thick-walled steel tube (diameter: $150 \mathrm{~mm}$, thickness: $30 \mathrm{~mm}$, and height: $800 \mathrm{~mm}$ ) and two upper/lower flange covers. The form of circular micro spray was used in the agitation mode. In this way, the reaction system was mixed evenly and promoted the reaction. The low temperature of the reactor was provided by the cooling medium of the constant temperature bath. The whole reactor was placed in a lowtemperature bath. Two temperature sensors were set in the reactor and the bath tank for measuring and controlling the reaction temperature. High-pressure gas from the gas bottle was released in a buffer tank (effective volume $20 \mathrm{~L}$ ) before the hydrate formation for maintaining the stability of the entrained flow reactor and preventing dangerous gas reflux.

2.2. Measuring Instruments. The pressure of the reactor was measured by a pressure transducer. MPM480 explosionproof type of pressure transmitter was selected from Shaanxi Mike sensor Co., Ltd. Its highest working pressure was $100 \mathrm{MPa}$, with an accuracy of $\pm 0.25 \%$ FS. The temperature was measured by a sheathed platinum resistance thermometer (model WZPK2103). Then it was transformed into a digital signal by a platinum resistance temperature transmitter, which was transported to the controller to be recorded and displayed.

2.3. Experimental Materials. In the present analysis, deionized water and 99.9\% methane gas (Beijing AP BAIF Gases Industry Co., Ltd., Beijing, China) were used. The surfaceactive agent used in the experiment was sodium dodecyl sulfate (SDS), and the purity was $99 \%$. SDS solutions were prepared at $300 \mathrm{ppm}(\mathrm{mg} / \mathrm{kg})$ by the deionized water. All chemicals were used as received. All other chemicals were used without further purification.

2.4. Calculation. The moles of gas (methane) consumed in hydrate and rate of hydrate formation were calculated using the recorded pressure and temperature data during the period of experiment.

2.4.1. Moles of Gas Consumption. The consumption of moles of gas during hydrate formation has been calculated using

$$
\Delta n_{H}=V_{g}\left(\frac{P_{i}}{Z_{i} R T}-\frac{P_{t}}{Z_{t} R T}\right)
$$

where $\Delta n_{H}$ is the moles of methane consumed at time, $t, V_{g}$ is the volume of the gas inside the reactor, $P_{i}$ is the pressure at the start of hydrate formation, $Z_{i}$ is the compressibility factor at the start of hydrate formation, $R$ is the ideal gas constant, $T$ is the average temperature of the gas during hydrate formation experiment, $P_{t}$ is the pressure of the reactor at $t$, and $Z_{t}$ is the compressibility factor of the gas in the reactor at t. $Z_{i}$ and $Z_{t}$ are calculated using Pitzer correlation (Smith et al., 2001). The moles of methane consumed $\left(\Delta n_{H}\right)$ per mole of water $\left(n_{w}\right)$ are calculated using

$$
N G_{t}=\Delta n_{\downarrow}(\mathrm{mol} / \mathrm{mol})=\frac{\Delta n_{H}}{n_{w}} .
$$


TABLE 1: Experimental parameters for hydrate formation.

\begin{tabular}{lcccc}
\hline Pressure/MPa SDS/ppm & $\begin{array}{c}\text { Reaction } \\
\text { time/min }\end{array}$ & $\begin{array}{c}\text { Gas storage } \\
\mathrm{mmol} / \mathrm{mol}\end{array}$ & $\begin{array}{c}\text { Reaction } \\
\text { rate } \\
\mathrm{mmol} / \mathrm{min}\end{array}$ \\
\hline 6 & 300 & 265 & 145.97 & 0.07 \\
6 & 0 & 445 & 97.31 & 0.03 \\
7 & 0 & 209 & 154.55 & 0.12 \\
\hline
\end{tabular}

2.4.2. Rate of Hydrate Formation. The rate of methane hydrate formation has been calculated using a discrete forward difference method as shown in

$$
\frac{d N_{t}}{d t}=\left(\frac{d \Delta n_{H}}{d t}\right)_{t}=\frac{\Delta n_{H, t+\Delta t}-\Delta n_{H, t}}{\Delta t}
$$

where $\Delta t$ is the time difference between two observations and the average rate of methane hydrate formation was calculated every $60 \mathrm{~min}$ and plotted.

2.5. Experimental Processes. First of all, the experimental gas was injected into the reactor with a water filled reactor until the remaining volume of the reaction was required. Then the gas supply booster pump started, and the freezing unit began to cool the reaction kettle. When the pressure and temperature were stable, the circulating pump was opened for the hydrate formation process. As the reaction was carried out, the consumption of the gas was automatically added by the booster pump to ensure that the pressure was constant during the reaction. When the pressure and temperature were constant, the pressure relief operation can be carried out. Finally, the formation of the hydrate was removed to determinate the gas storage and other physical and chemical indicators. The experiment data was automatically detected and recorded by the control display system.

In this paper, three experiments were conducted to determine the SDS and the pressure factor. The amount of water in the reaction was $2.0 \mathrm{~L}$. Specific experimental conditions were shown in Table 1.

\section{Experimental Results and Discussion}

3.1. Consumption of Gas and Temperature. The hydrate formation process is an exothermic reaction and usually experiences three stages: induction stage, rapid reaction stage, and ending stage. If the hydrate formed in a short time, the reaction heat cannot be transferred in time which results in rapid rise of the temperature in the reactor.

Figure 2 shows the temperature and the moles of gas consumed per mole of water variation during methane hydrate formation process in the presence of $300 \mathrm{ppm}$ SDS aqueous solution at 6.0 MPa. In the course of hydrate formation, the high-pressure reactor is a semiclosed system. In the reactor, methane is continuously added as a result of the hydrate formation process, in order to ensure the reaction under constant pressure. At 6.0 $\mathrm{MPa}$, the temperature and gas consumption of the whole process of gas hydrate nucleation and crystal growth are shown in Figure 2. The three stages

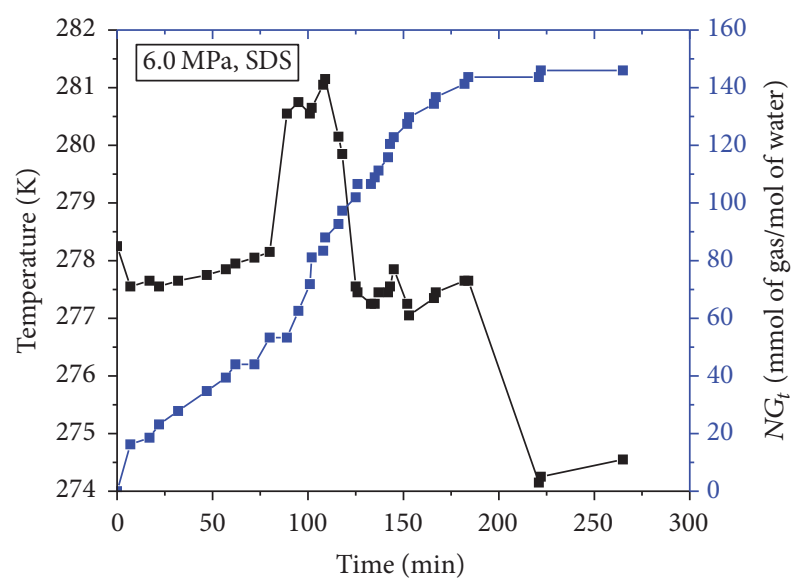

FIgURE 2: Hydrate formation in $300 \mathrm{ppm}$ SDS solution at $6.0 \mathrm{MPa}$.

of the formation of the hydrate formation process are clearly shown in the figure, that is, induction stage, rapid reaction stage, and ending stage.

In the induction stage, the gas consumption growth rate is slow, and there was a basic linear rise. Temperature is basically kept constant, which shows that the reaction heat is released in time. It is obviously shown in Figure 2 that the temperatures rise by about $0.5 \mathrm{~K}$ after $80 \mathrm{~min}$ of the high-pressure gaseous methane entering into the cool cell. At the rapid reaction stage, the gas consumption has increased sharply. Due to the rapid reaction rate, the heat produced by the reaction cannot be released in time and the temperature in the reactor increased. The system temperatures rise by $3.3 \mathrm{~K}$ secondly between 80 and $180 \mathrm{~min}$ of the gas intake process being completed, which indicates that lots of methane hydrates form during this period. The gas consumption growth rate increased evidently. In the ending stage, the amount of gas consumption became smaller and gradually reached zero, the temperature dropped sharply, and the reaction stopped.

Figures 3 and 4 show the moles of gas consumed per mole of water and temperature during methane hydrate formation in the presence of SDS and pure water. As shown in Figures 3 and 4 , the different aqueous solution and pressures of the systems are of similar morphology which still have the three stages. The experiments conducted at $300 \mathrm{ppm}$ SDS aqueous solution show higher moles of gas consumption per mole of water as compared with pure water (see Figure 4) at 6.0 MPa. But the temperature change is relatively stable. The experiment conducted at $300 \mathrm{ppm}$ of SDS aqueous solution at 7.0 MPa shows the highest moles of gas consumption per mole of water as compared with $300 \mathrm{ppm}$ of SDS aqueous solutions and pure water (see Figures 3 and 4 ) at $6.0 \mathrm{MPa}$, while the change of temperature in pure water at $6.0 \mathrm{MPa}$ (shown in Figure 4) is smoother than that of two other reaction conditions.

The comparison between Figures 3 and 4 can be seen: the amount of gas consumption in the pure water in the nucleation stage is smaller but the time is longer than that of SDS aqueous solution. In the rapid reaction stage, the rate of reaction is more rapid in SDS solution and the temperature of the 


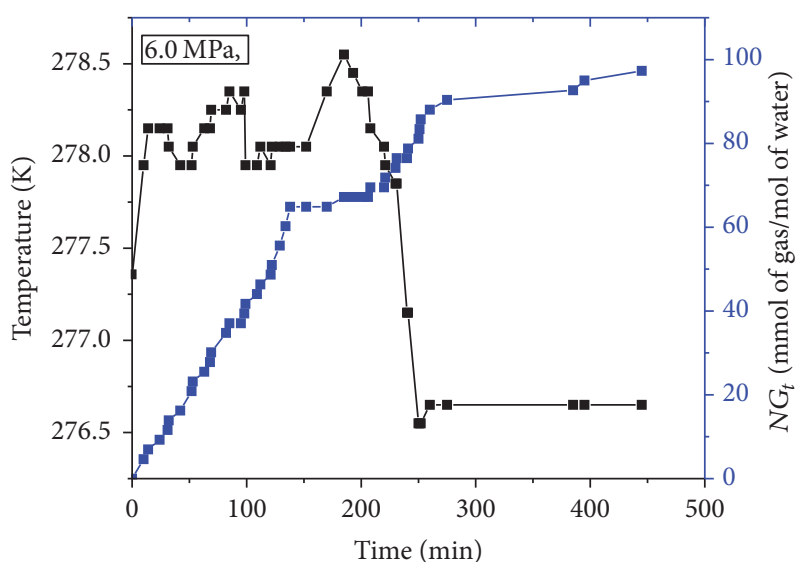

Figure 3: Hydrate formation in pure water at 6.0 MPa.

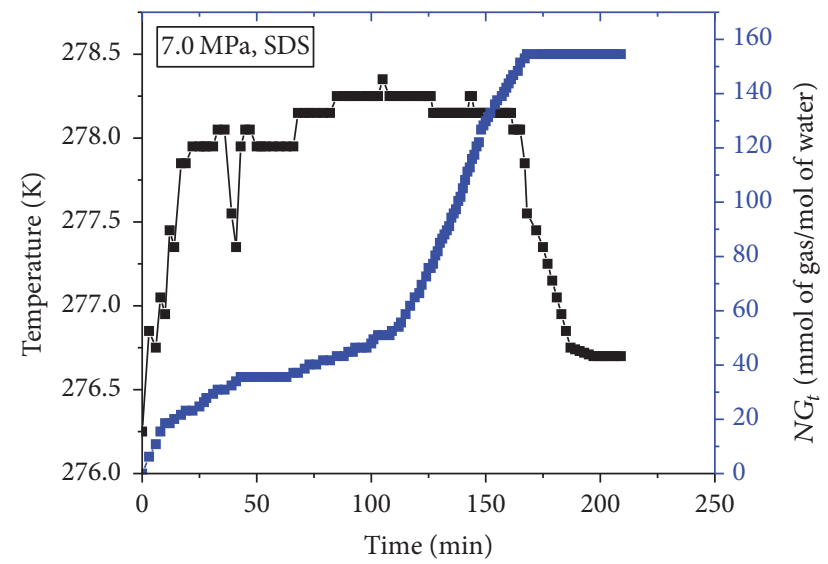

FIgURE 4: Hydrate formation in $300 \mathrm{ppm}$ SDS solution at 7.0 MPa.

reactor increases to $281 \mathrm{~K}$, while in pure water the temperature is relatively stable. From the whole process of the reaction of gas and pure water, nucleation induction stage is relatively long, but the rapid reaction stage is short and gas consumption is less. The surfactant SDS solution can provide more interface reactions, dominant heterogeneous nucleation, and fast reaction speed.

Figure 5 shows comparison of the gas consumptions for the selected best sets of hydrate system at 7.0 and 6.0 MPa. It has been observed that the hydrate system containing pure water with SDS (300 ppm) shows higher cumulative moles of gas consumption per mole of water as compared with the pure water systems. One of the distinct advantages of using SDS is that it lowers the induction time as compared to pure water by achieving faster nucleation. Through the experiment, it can be found that the contact area of gas and water can be greatly improved by using the spray atomization to generate hydrate. Therefore, the effect of the spray atomization method is better than that in the promotion of the rate of hydrate preparation.

3.2. Rate of Hydrate Formation. Figure 6 shows the rate of methane hydrate formation at $300 \mathrm{ppm}$ SDS aqueous solution

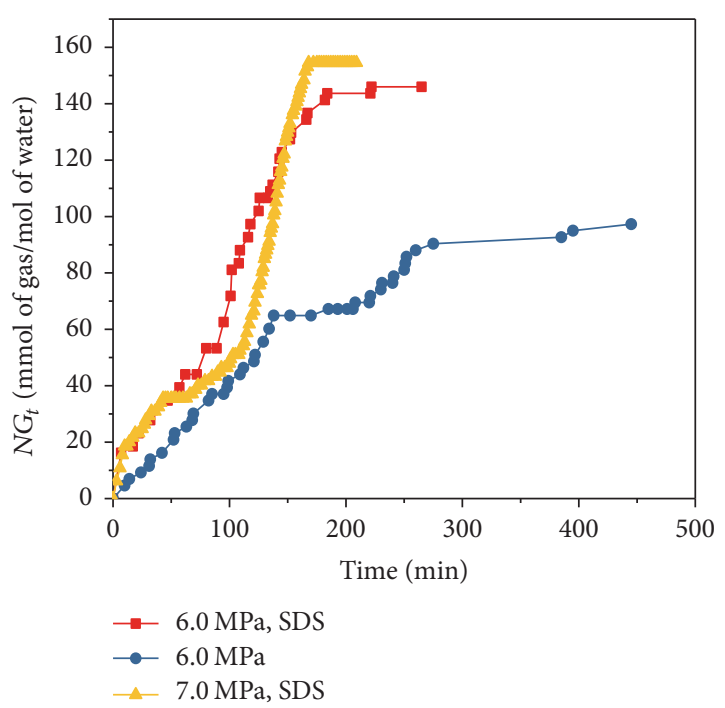

FIGURE 5: Methane consumed per mole of water in different condition.



FIGURE 6: Rate of hydrate formation in different condition.

in comparison with pure water at 7.0 and 6.0 MPa. It has been observed that, for the initial hours of the hydrate formation experiment, system with SDS maintained the highest rate of hydrate formation followed by pure water.

Overall, SDS aqueous solution has maintained relatively a high rate of hydrate formation as compared to pure water throughout the experiment. This indicates that the induction time using SDS has been reduced and the rate of hydrate formation has been favorable.

The hydrate formation rate is controlled by the pressure, temperature, and composition of the impact. In addition, the surface tension of the liquid has an important influence on the formation. Adding appropriate surfactants can greatly promote the crystallization reaction. 


\section{Conclusions}

Gas hydrate formation is the process of gas-liquid reaction in the formation of gas-liquid phase interface. The process is affected by the formation conditions, heat transfer, and the intrinsic characteristics of the reaction. The reaction rate can be improved by the experiments and measurement, and the induction period of the reaction can be greatly reduced. There are two reasons for this: (1) the contact surface is increased, so the reaction is carried out; (2) the reaction heat is rapidly spreading out. Gas hydrate is proved to be synthesized by spray atomization, and the high efficiency can be realized by artificial means. Thus, gas storage, separation, transportation, and so on can be reached through the hydrate. As spray atomization factors of hydrate preparation, the thermodynamic process involves contact heat and mass transfer, induction time, and nucleation. The study of these factors will have an important role in the development of gas hydrate storage and transportation technology.

\section{Conflicts of Interest}

The authors declare that there are no conflicts of interest regarding the publication of this paper.

\section{Acknowledgments}

The financial supports were received from Research Project Supported by Shanxi Scholarship Council of China (no. 2016-095), Fund Program for the Scientific Activities of Selected Returned Overseas Professionals in Shanxi Province (no. 201697), Fund Program for the Scientific Activities of Selected Returned Overseas Professionals in MOHRSS (no. 2015192), and Doctoral Project of Taiyuan University of Science and Technology of Shanxi Province in China (Grant no. 20122003). Thanks are due to Collaborative Innovation Center of Taiyuan Heavy Machinery Equipment and Shanxi Provincial Key Laboratory of Metallurgical Device Design Theory and Technology.

\section{References}

[1] E. D. Sloan and C. A. Koh, Clathrate Hydrates of Natural Gases, CRC Press, Taylor \& Francis Group, New York, NY, USA, 3rd edition, 2008.

[2] C. A. Koh, E. D. Sloan, and A. Sum, Natural Gas Hydrates in Flow Assurance, Gulf Professional Publishing, Burlington, NJ, USA, 2011.

[3] X.-S. Li, H. Zhan, C.-G. Xu, Z.-Y. Zeng, Q.-N. Lv, and K.F. Yan, "Effects of tetrabutyl-(ammonium/phosphonium) salts on clathrate hydrate capture of $\mathrm{CO}_{2}$ from simulated flue gas," Energy and Fuels, vol. 26, no. 4, pp. 2518-2527, 2012.

[4] M. Yang, W. Jing, J. Zhao, Z. Ling, and Y. Song, "Promotion of hydrate-based $\mathrm{CO}_{2}$ capture from flue gas by additive mixtures (THF (tetrahydrofuran) + TBAB (tetra-n-butyl ammonium bromide))," Energy, vol. 106, pp. 546-553, 2016.

[5] P. Babu, R. Kumar, and P. Linga, "Pre-combustion capture of carbon dioxide in a fixed bed reactor using the clathrate hydrate process," Energy, vol. 50, no. 1, pp. 364-373, 2013.
[6] Y. Zhao, J. Zhao, D. Shi, Z. Feng, W. Liang, and D. Yang, "MicroCT analysis of structural characteristics of natural gas hydrate in porous media during decomposition," Journal of Natural Gas Science and Engineering, vol. 31, pp. 139-148, 2016.

[7] J. Zhao, Y. Zhao, and W. Liang, "Hydrate-based gas separation for methane recovery from coal mine gas using tetrahydrofuran," Energy Technology, vol. 4, no. 7, pp. 864-869, 2016.

[8] D.-L. Zhong, Z. Li, Y.-Y. Lu, J.-L. Wang, and J. Yan, "Evaluation of $\mathrm{CO}_{2}$ removal from a $\mathrm{CO}_{2}+\mathrm{CH}_{4}$ gas mixture using gas hydrate formation in liquid water and THF solutions," Applied Energy, vol. 158, pp. 133-141, 2015.

[9] P. Linga and M. A. Clarke, "A review of reactor designs and materials employed for increasing the rate of gas hydrate formation," Energy \& Fuels, vol. 31, no. 1, pp. 1-13, 2017.

[10] Y. Zhong and R. E. Rogers, "Surfactant effects on gas hydrate formation," Chemical Engineering Science, vol. 55, no. 19, pp. 4175-4187, 2000.

[11] K. Yoshikawa, K. Yuichi, and K. Takahiro, "Production method for hydrate and device for proceeding the same," United States Patent: 524753, 2000.

[12] D. Kashchiev and A. Firoozabadi, "Nucleation of gas hydrates," Journal of Crystal Growth, vol. 243, no. 3-4, pp. 476-489, 2002.

[13] J.-Y. Liu, J. Zhang, Y.-L. Liu, X.-H. Tan, and J. Zhang, "Experimental and modeling studies on the prediction of gas hydrate formation," Journal of Chemistry, vol. 2015, Article ID 198176, 5 pages, 2015.

[14] S. Chang-yu, C. Guang-jin, and G. Tian-min, "The status of the kinetics of hydrate nucleation," Acta Petrolei Sinica, vol. 22, no. 4, pp. 82-86, 2001 (Chinese).

[15] Y. H. Mori, "Estimating the thickness of hydrate films from their lateral growth rates: application of a simplified heat transfer model," Journal of Crystal Growth, vol. 223, no. 1-2, pp. 206-212, 2001.

[16] N. Gnanendran and R. Amin, "Modelling hydrate formation kinetics of a hydrate promoter-water-natural gas system in a semi-batch spray reactor," Chemical Engineering Science, vol. 59, no. 18, pp. 3849-3863, 2004. 



Carbohydrate Chemistry



The Scientific World Journal
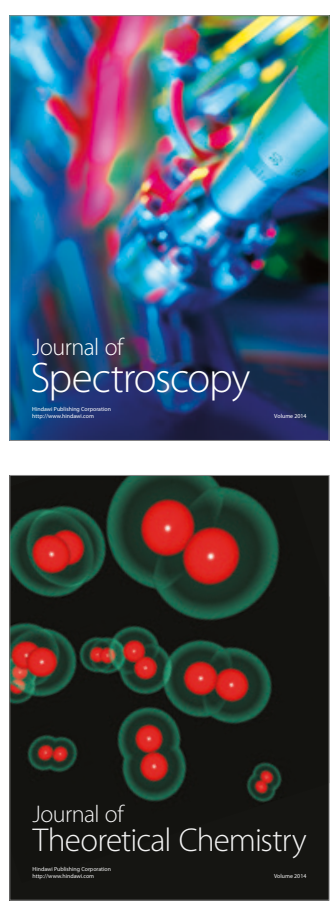
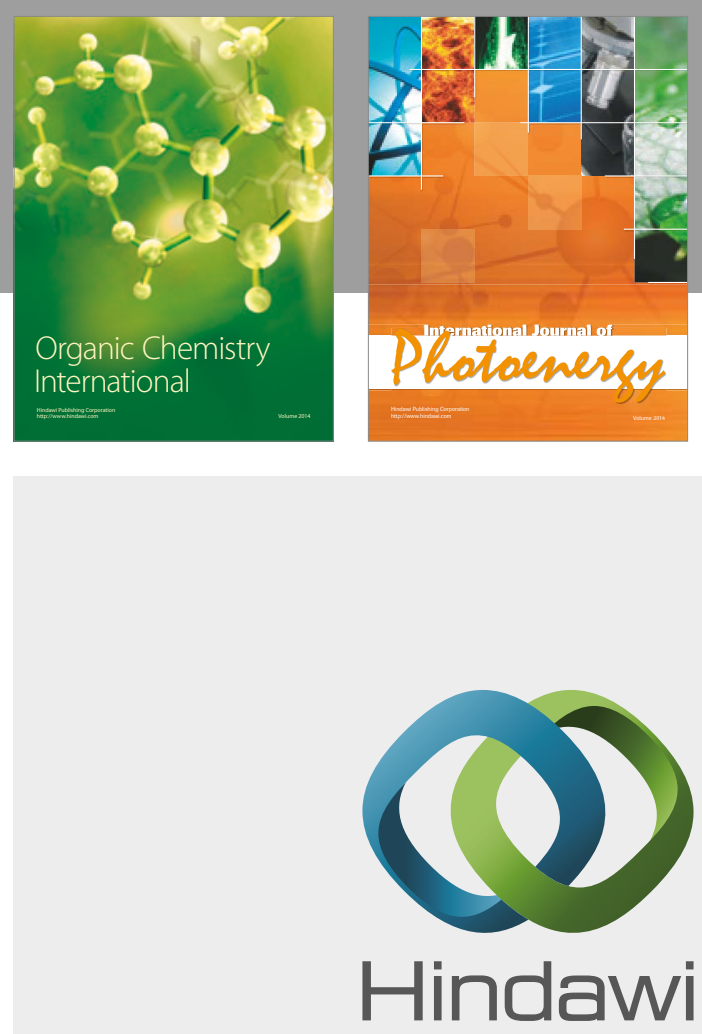

Submit your manuscripts at

https://www.hindawi.com

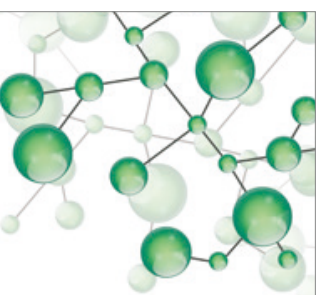

International Journal of

Inorganic Chemistry

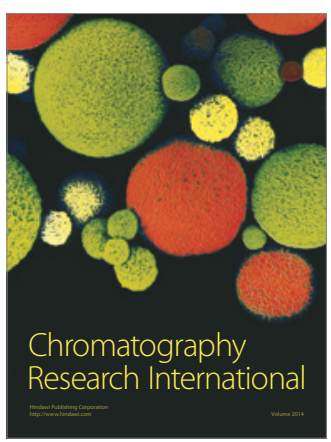

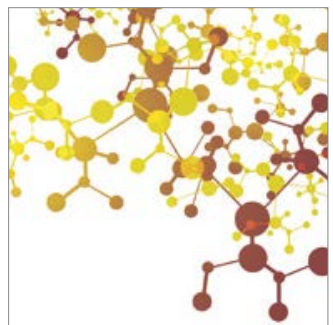

Applied Chemistry
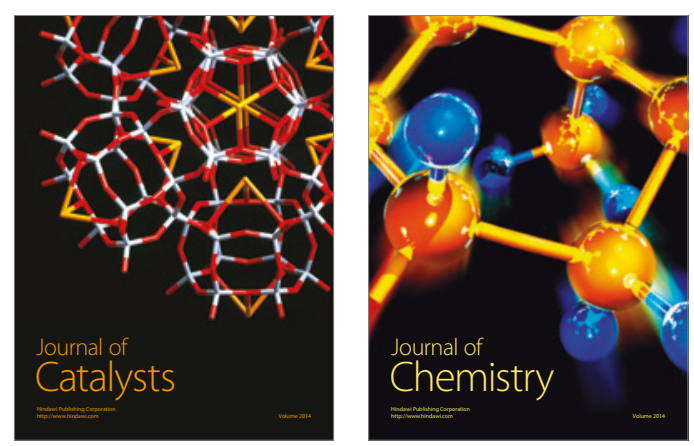
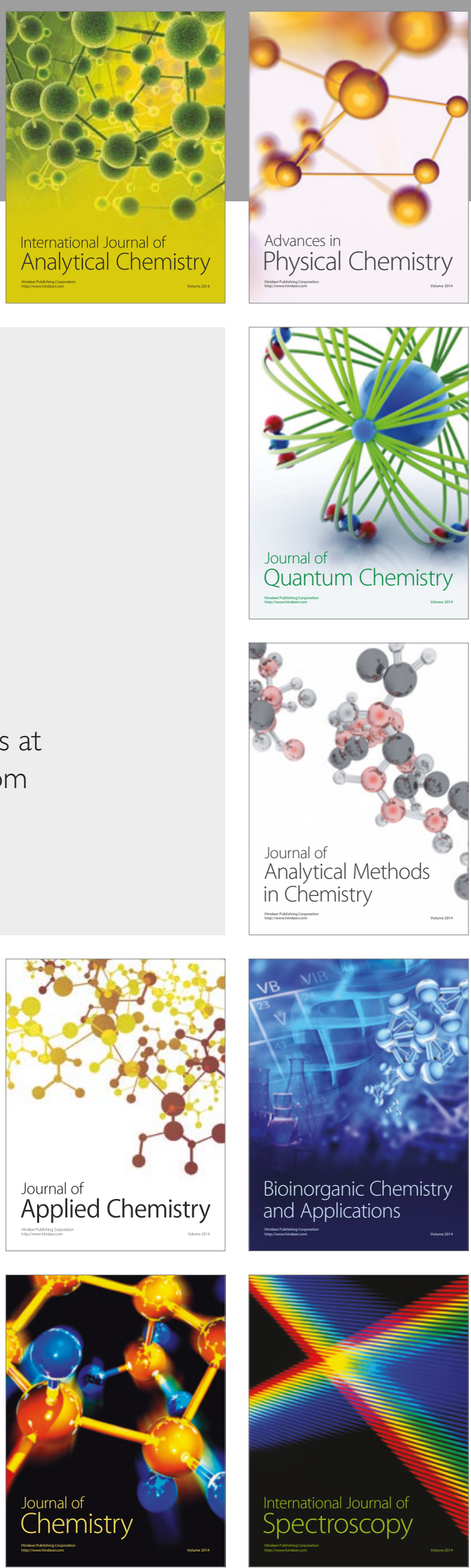